\title{
Consideraciones técnicas para colecistectomía laparoscópica en paciente con situs inversus totalis: presentación de caso y revisión de la literatura
}

\author{
Technical considerations for laparoscopic cholecystectomy in a patient with situs \\ inversus totalis: case report and review of the literature
}

José D. Hernández-Marín ${ }^{*}$ e Hitandéhuitl Guevara-Valerio²

${ }^{1}$ Servicio de Cirugía General, Hospital General de Zona con Medicina Familiar \#8 Dr. Gilberto Flores Izquierdo, Instituto Mexicano del Seguro Social (IMSS); ${ }^{2}$ Servicio de Anestesiología, Hospital General Regional \#2, IMSS. Ciudad de México, México

\begin{abstract}
Resumen
El situs inversus es una rara anomalía congénita. El primer caso de colecistectomía laparoscópica en un paciente con situs inversus totalis se realizó en 1991. Se presenta el caso de un paciente varón, de 66 años, que presentó litiasis vesicular. Durante el protocolo preoperatorio se documentó situs inversus totalis. Fue intervenido quirúrgicamente de forma electiva y se realizó colecistectomía laparoscópica sin complicaciones. Diversos autores reportan casos en los que realizaron colecistectomía laparoscópica con tres y cuatro trócares, y con diferente posición de los puertos. Es técnicamente demandante realizar una colecistectomía laparoscópica en pacientes con situs inversus totalis, pero es seguro realizarla.
\end{abstract}

Palabras clave: Situs inversus. Colecistectomía. Laparoscopia.

\begin{abstract}
Situs inversus totalis is a rare congenital anomaly. The first case of laparoscopic cholecystectomy in a patient with situs inversus totalis was reported in 1991. A 66-year-old male with cholelithiasis. Clinical assessment and radiological investigation diagnosed situs inversus totalis. Laparoscopic cholecystectomy was performed electively without complications. Authors report changes in the number and position of trocars to perform laparoscopic cholecystectomy. Although it is technically challenging, it is safe to perform a laparoscopic cholecystectomy in a patient with situs inversus totalis.
\end{abstract}

Key words: Situs inversus. Laparoscopy. Cholecystectomy.

\section{Antecedentes}

El situs inversus totalis es una rara anomalía congénita caracterizada por la transposición de los órganos torácicos y abdominales al otro lado del cuerpo. Tiene una incidencia de 1:10,000-20,0001,2. Existen otras anomalías anatómicas en las que la vesícula biliar se encuentra del lado izquierdo del cuerpo. Se denomina sinistroposición cuando la vesícula se localiza al lado

\section{Correspondencia:}

*José D. Hernández Marín

Avda. División del Norte, 3395-409

El Rosario, Del. Coyoacán

Fecha de recepción: 16-01-2020

Cir Cir. 2020;88(S2):5-8

C.P. 04380, Ciudad de México, México

Fecha de aceptación: 07-06-2020

Contents available at PubMed

E-mail: dr.davidhm@outlook.com

DOI: $10.24875 / C I R U .20000038$

www.cirugiaycirujanos.com

0009-7411/@ 2020 Academia Mexicana de Cirugía. Publicado por Permanyer. Este es un artículo open access bajo la licencia CC BY-NC-ND (http://creativecommons.org/licenses/by-nc-nd/4.0/). 
izquierdo del ligamento redondo y debajo del lóbulo hepático izquierdo sin situs inversus ${ }^{3,4}$.

El primer caso de colecistectomía laparoscópica en un paciente con situs inversus totalis fue reportado en 19915; desde entonces se han reportado 91 casos en la literatura ${ }^{6}$. En México se reportó un caso de coledocolitiasis resuelto con cirugía abierta ${ }^{7}$.

\section{Caso clínico}

Varón de 66 años con antecedente de hipertensión arterial sistémica. Inició su padecimiento en agosto de 2018 con dolor epigástrico. Se realizó ultrasonido abdominal que reportó litiasis vesicular y situs inversus. Durante el protocolo preoperatorio se realizó tomografía abdominal que documentó situs inversus totalis y litiasis vesicular. Los resultados de laboratorio reportaron pruebas de función hepática sin alteraciones. En abril de 2019 fue intervenido quirúrgicamente de forma electiva. Se realizó colecistectomía laparoscópica sin complicaciones. Egresó al día siguiente de la cirugía (Figs. 1 y 2).

\section{Discusión}

AlKhlaiwy, et al. ${ }^{6}$ reportaron un caso en el que utilizaron tres trócares de $5 \mathrm{~mm}$ : uno en el hipocondrio izquierdo como puerto de trabajo, uno subxifoideo para auxiliarse y uno en el flanco izquierdo para el ayudante, además de un puerto óptico umbilical de $11 \mathrm{~mm}$.

Alam y Santra ${ }^{8}$ utilizaron cuatro trócares: dos de $10 \mathrm{~mm}$ (infraumbilical y subxifoideo) y dos de $5 \mathrm{~mm}$ (hipocondrio y flanco izquierdos). Utilizaron el puerto subxifoideo para disecar y el puerto en el hipocondrio izquierdo para traccionar la bolsa de Hartmann.

Ren, et al. ${ }^{9}$ reportaron un caso en el que utilizaron cuatro puertos: uno infraumbilical de $12 \mathrm{~mm}$, uno subxifoideo de $10 \mathrm{~mm}$ y dos de $5 \mathrm{~mm}$ (hipocondrio y flanco izquierdos). Utilizaron el puerto subxifoideo para disecar y el puerto en el hipocondrio izquierdo para traccionar la bolsa de Hartmann. Alsabek, et al. ${ }^{2}$ utilizaron la misma técnica, y vale la pena mencionar que el cirujano era zurdo.

Raghuveer, et al. ${ }^{10}$ utilizaron cuatro trócares: infraumbilical y subxifoideo de $10 \mathrm{~mm}$, hipocondrio y flanco izquierdos de $5 \mathrm{~mm}$. El cirujano disecó el triángulo de Callot con la mano derecha desde el puerto en el hipocondrio izquierdo y el ayudante traccionó la bolsa de Harmann. Arya, et al. ${ }^{11}$ y Hall, et al. ${ }^{12}$ realizaron la cirugía de la misma forma.

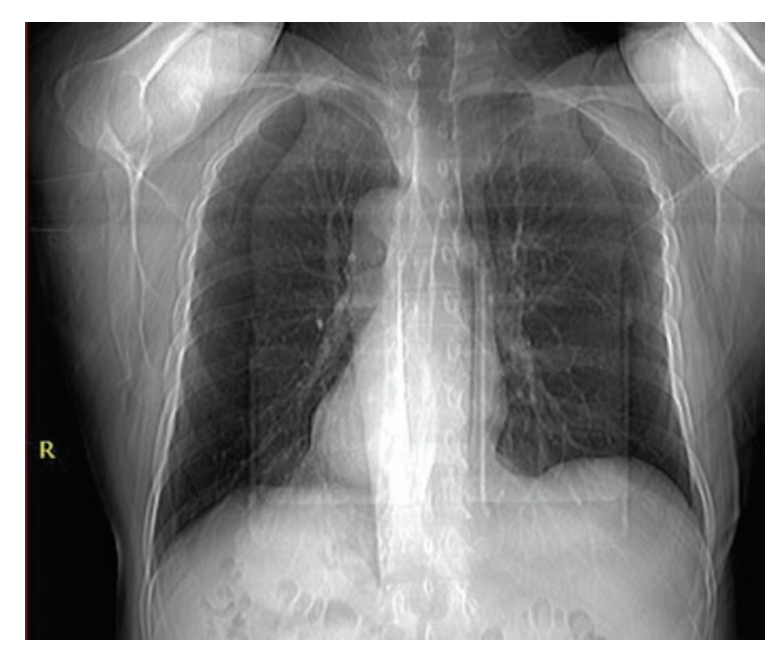

Figura 1. Radiografía de tórax en la que se observa dextrocardia.

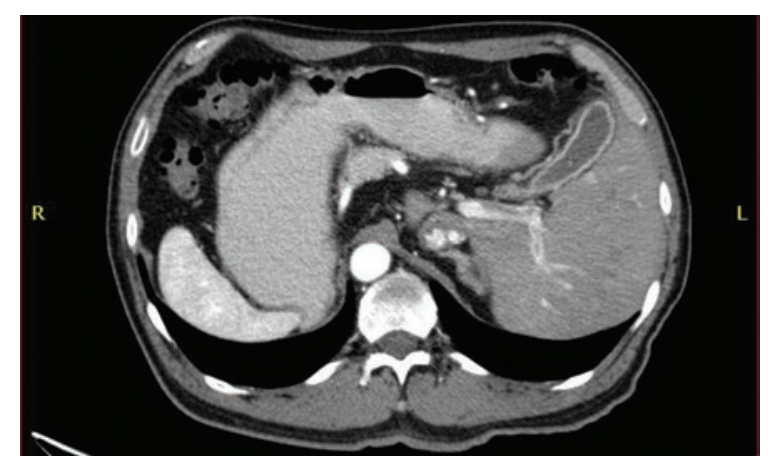

Figura 2. Tomografía abdominal que evidencia el hígado y la vesícula biliar en el hemiabdomen izquierdo.

Ali y Attash ${ }^{13}$ colocaron dos trócares de $10 \mathrm{~mm}$ infraumbilical y en el hipocondrio izquierdo, y dos trócares de $5 \mathrm{~mm}$ en el hipocondrio derecho y el flanco izquierdo. Utilizaron el trócar en el hipocondrio derecho para traccionar la bolsa de Hartmann y el trócar en el hipocondrio izquierdo para disecar.

En México, Melchor-González, et al.7 reportaron el caso de una paciente con coledocolitiasis que se resolvió con exploración de vía biliar abierta.

\section{Conclusiones}

Utilizamos cuatro puertos: supraumbilical e hipocondrio izquierdo con trócares de $10 \mathrm{~mm}$, subxifoideo y en el flanco izquierdo con trócares de $5 \mathrm{~mm}$. El cirujano traccionó la bolsa de Hartmann a través del puerto subxifoideo y realizó la disección del triángulo de Calot a través del puerto en el hipocondrio izquierdo, y el ayudante traccionó el fondo a través 


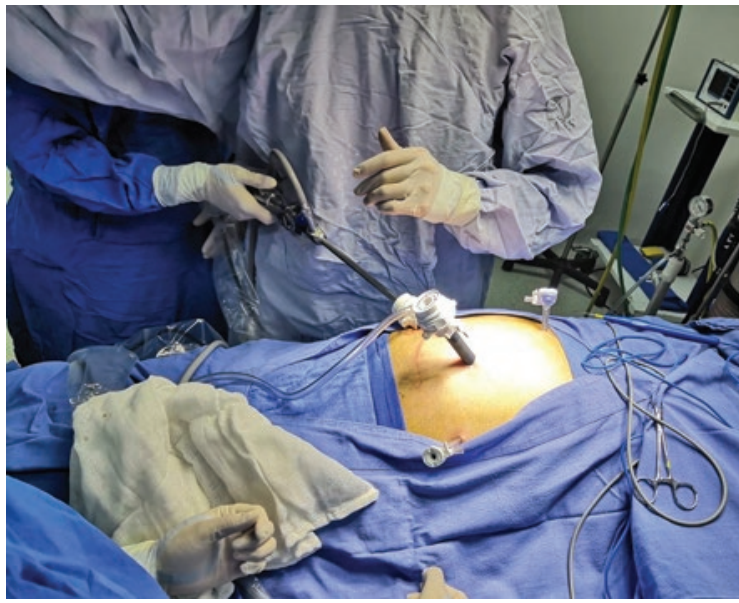

Figura 3. Imagen en la que se observa la colocación de los trócares. En el centro se encuentra el puerto de trabajo colocado en el hipocondrio izquierdo.

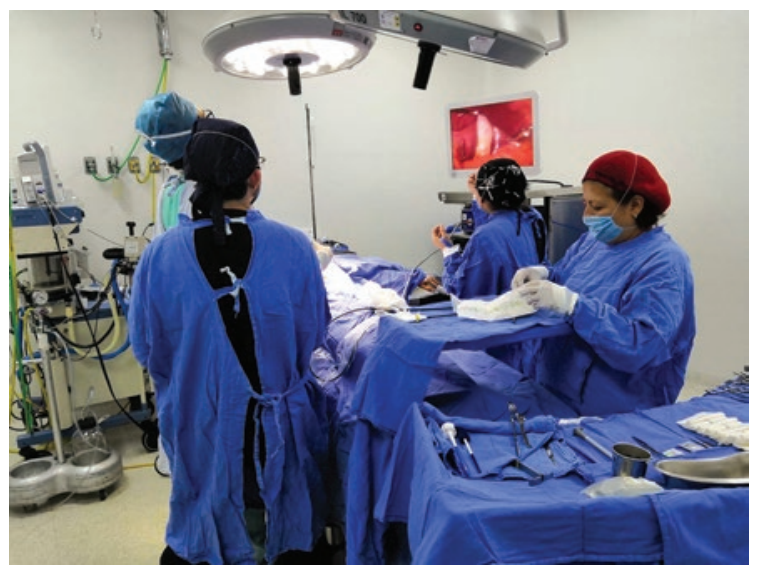

Figura 4. Imagen en la que se observa la colocación del equipo quirúrgico: el cirujano y el primer ayudante del lado derecho, y el segundo ayudante y la instrumentista del lado izquierdo.

del puerto en el flanco izquierdo. Al obtener una adecuada visión crítica, se colocaron los clips en el conducto y la arteria cística. Se disecó la vesícula del lecho y se extrajo a través del puerto supraumbilical (Figs. 3 a 6 ).

No existe una técnica establecida para realizar la colecistectomía laparoscópica en pacientes con situs inversus. En nuestro caso utilizamos cuatro puertos, con el puerto de trabajo en el hipocondrio izquierdo.

Es técnicamente demandante realizar una colecistectomía laparoscópica en pacientes con situs inversus totalis, pero es seguro hacerla si se siguen los pasos para obtener la visión crítica.

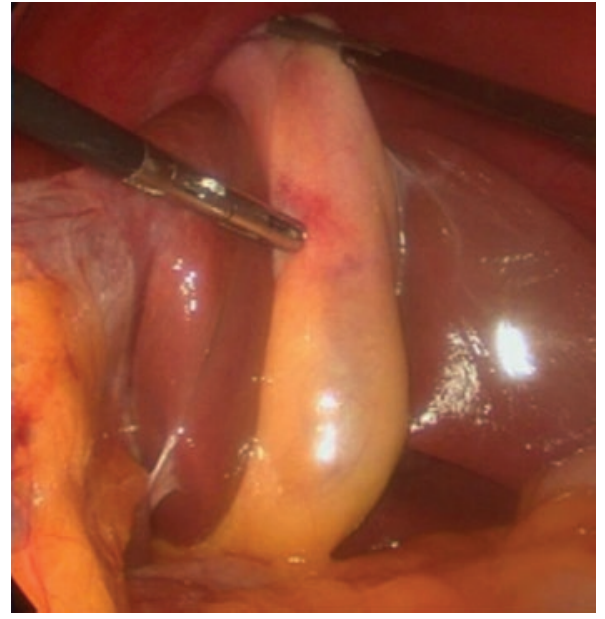

Figura 5. Imagen intraabdominal al traccionar la vesícula del fondo.

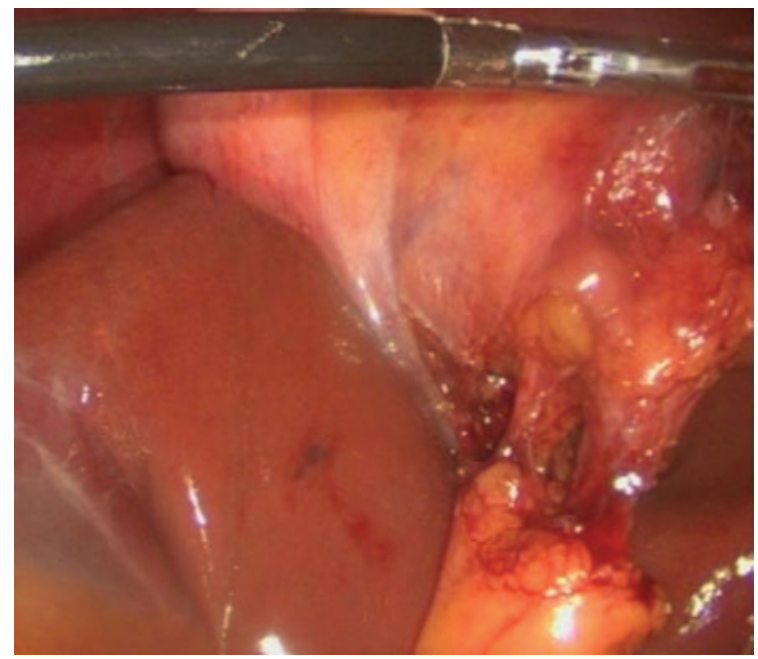

Figura 6. Imagen intraabdominal en la que se observa la visión crítica.

\section{Conflicto de intereses}

Los autores manifiestan no tener conflicto de intereses alguno.

\section{Responsabilidades éticas}

Protección de personas y animales. Los autores declaran que para esta investigación no se han realizado experimentos en seres humanos ni en animales.

Confidencialidad de los datos. Los autores declaran que han seguido los protocolos de su centro de trabajo sobre la publicación de datos de pacientes. 
Derecho a la privacidad y consentimiento informado. Los autores han obtenido el consentimiento informado de los pacientes y/o sujetos referidos en el artículo. Este documento obra en poder del autor de correspondencia.

\section{Bibliografía}

1. Salama IA, Abdullah MH, Houseni M. Laparoscopic cholecystectomy in situs inversus totalis: feasibility and review of the literature. Int J Surg Case Reports. 2013;4:711-5.

2. Alsabek MB, Arafat S, Aldirani A. A case report of laparoscopic cholecystectomy in situs inversus totalis: technique and anatomical variation. Int J Surg Case Reports. 2016;28:124-6.

3. Nguyen TH, Nguyen TS, Van Nguyen PD, Dang TN, Talarico EF Jr. Sinistroposition: a case report of true left-sided gallbladder in a Vietnamese patient. Int J Surg Case Reports. 2018;51:82-5.

4. Abongwa HK, DeSimone B, Alberici L, laria M, Perrone G, Tarasconi A, et al. Implications of left-sided gallbladder in the emergency setting: retrospective review and top tips for safe laparoscopic cholecystectomy. Surg Laparosc Endosc Percutan Tech. 2017;27:220-7.
5. Campos L, Sipes E. Laparoscopic cholecystectomy in a 39-year-old female with situs inversus. J Laparoendosc Surg. 1991;1:123-5.

6. AlKhlaiwy O, AIMuhsin AM, Zakarneh E, Taha MY. Laparoscopic cholecystectomy in situs inversus totalis: case report with review of techniques. Int J Surg Case Reports. 2019;59:208-12.

7. Melchor-González JM, Pérez-García R, Torres-Vista M, Rodríguez-Brambila VR. Situs inversus, reporte de dos casos. Cir Cir. 2000;68:72-5.

8. Alam A, Santra A. Laparoscopic cholecystectomy in a case of situs inversus totalis: a review of technical challenges and adaptations. Ann Hepatobiliary Pancreat Surg. 2017;21:84-7.

9. Ren J, Li S, Geng Y, Xiao R. Modified laparoscopic cholecystectomy technique for treatment of situs inversus totalis: a case report. J Int Med Res. 2017;45:1261-7.

10. Raghuveer MN, Manesh SS, Sunil Kumar BB. Laparoscopic cholecystectomy in situs inversus totalis. J Clin Diagn Res. 2014;8:ND03-5.

11. Arya SV, Das, A, Singh S, Kalwaniya DS, Sharma A, Thukral BB. Technical difficulties and its remedies in laparoscopic cholecystectomy in situs inversus totalis: a rare case report. Int J Surg Case Reports. 2013;4:727-30.

12. Hall TC, Barandiaran J, Perry EP. Laparoscopic cholecystectomy in situs inversus totalis: is it safe? Ann R Coll Surg Engl. 2010;92:W30-2.

13. Ali MS, Attash SM. Laparoscopic cholecystectomy in a patient with situs inversus totalis: a case report with review of the literature. BMJ Case Rep. 2013;2013:bcr2013201231. 